\title{
枞酸和松香在紫外光辐照下的氧化反应动力学
}

\author{
刘雄民* 秦荣秀 黄品鲜 刘珈伶 马 丽 李伟光 \\ (广西大学化学化工学院, 南宁 530004)
}

\begin{abstract}
摘要：设计了紫外光辐照下枞酸和松香的氧化反应装置, 采用紫外分光光度法实时测定它们的氧化反应过程, 计算氧化反应动力学常数 $\left(k_{\mathrm{b}}\right)$ 和反应活化能 $\left(E_{\mathrm{a}}\right)$, 考察光量子产率 $(\Phi)$ 和光强度 $(I)$ 对氧化反应动力学的影响. 结果 表明, 枞酸和松香的光氧化反应均呈表观一级反应, 它们的速率常数的对数与光强度的对数均呈线性关系. 枞酸 在 20,25 和 $35{ }^{\circ} \mathrm{C}$ 下的关系式分别为: $\ln k_{\mathrm{b}}=0.9911 \ln I-8.860, \ln k_{\mathrm{b}}=0.8786 \ln I-8.069$ 和 $\ln k_{\mathrm{b}}=0.8364 \ln I-7.690$. 松香于 $20{ }^{\circ} \mathrm{C}$ 的关系式为: $\ln k_{\mathrm{b}}=1.204 \ln I-10.49$. 枞酸初始反应的光量子产率 $\Phi$ 为 0.471 . 且枞酸在不同光强度下的活化 能与光强度的对数成线性关系, 线性方程为: $E_{\mathrm{a}}=-7.549 \ln I+60.02$.
\end{abstract}

关键词: 枞酸; 松香; 光氧化反应; 动力学; 速率常数 中图分类号： O643; O644

\section{Oxidation Reaction Kinetics of Abietic Acid and Rosin under Ultraviolet Light Irradiation}

\author{
LIU Xiong-Min* QIN Rong-Xiu HUANG Pin-Xian LIU Jia-Ling MA Li LI Wei-Guang \\ (School of Chemistry and Chemical Engineering, Guangxi University, Nanning 530004, P. R. China)
}

\begin{abstract}
We designed an oxidation reactor for abietic acid and rosin oxidation under ultraviolet light irradiation. The oxidation process of abietic acid and rosin were determined by UV spectrophotometry and the rate constants $\left(k_{\mathrm{b}}\right)$ and activation energy $\left(E_{\mathrm{a}}\right)$ of the oxidation process were calculated. The kinetic effect of the quantum efficiency $(\Phi)$ and the light intensity $(I)$ on the photooxidation reaction were also investigated. The experimental results showed that the photooxidation kinetics of abietic acid and rosin were pseudo first order. The relationship between the natural logarithm of rate constant $\left(\ln k_{\mathrm{b}}\right)$ and the natural logarithm of the light intensity $(\ln I)$ is linear. When the reaction temperatures were 20,25 , and $35{ }^{\circ} \mathrm{C}$, the apparent reaction rate constants of abietic acid were: $\ln k_{\mathrm{b}}=0.9911 \ln I-8.860$, $\ln k_{\mathrm{b}}=0.8786 \ln I-8.069$, and $\ln k_{\mathrm{b}}=0.8364 \ln I-7.690$, respectively. At $20{ }^{\circ} \mathrm{C}$, the apparent reaction rate constant of rosin was $\ln k_{\mathrm{b}}=1.204 \ln I-10.49$. The initial quantum efficiency of abietic acid is 0.471 and the relationship between the activation energy $\left(E_{\mathrm{a}}\right)$ of abietic acid and the natural logarithm of the light intensity $(\ln I)$ is also linear: $E_{\mathrm{a}}=-7.549 \ln I+$ 60.02 .
\end{abstract}

Key Words: Abietic acid; Rosin; Photo-oxidation; Kinetics; Rate constant

松香是宝贵的可再生资源 ${ }^{[1]}$, 广泛应用于涂料 ${ }^{[2]}$ 、 油墨 ${ }^{[3]}$ 、胶粘剂 ${ }^{[4]}$ 、造纸施胶剂 ${ }^{[5]}$ 等领域, 由于这些产 品需要良好的耐候性, 因此, 对松香的光、热稳定性 和抗氧化性研究受到国内外关注. 松香的主要成分
是枞酸, 在热氧化研究方面, Harris ${ }^{[6]}$ 和王涛等 ${ }^{[7]}$ 指出 枞酸的自氧化首先发生在共轭双键的邻位碳上, 产 物为氢过氧化物及 1,2 -环氧衍生物, 本课题组 ${ }^{[8]}$ 研究 了松香和枞酸热氧化动力学, 得到它们的热氧化表

Received: April 8, 2010; Revised: April 19, 2010; Published on Web: June 29, 2010.

*Corresponding author. Email: xmliul@gxu.edu.cn; Tel: +86-771-3270732.

The project was supported by the National Key Technology R \& D Program of China (2007BAD82B01) and Science Foundation of Guangxi, China (0639001, 0832002).

国家科技支撑计划课题(2007BAD82B01)和广西科学基金项目(0639001, 0832002)资助

C. Editorial office of Acta Physico-Chimica Sinica 
观反应活化能分别为 80.2 和 $50.29 \mathrm{~kJ} \cdot \mathrm{mol}^{-1}$.

烯烃光化学和光敏氧化反应的研究 ${ }^{[0-12]}$ 表明, 共 轭烯烃容易发生分子内和分子间的双烯光环合加成 反应, 在有氧存在下, 共轭烯烃很容易发生 1,3-位加 成反应生成环内过氧化物, 环内过氧化物不稳定易 发生分解反应. Moore 等 ${ }^{[3-15]}$ 对松香中的几种成分 (左旋海松酸、长叶松酸、新松酸)的光氧化产物进行 系统研究, 其中左旋海松酸在乙醇溶液中添加敏化 剂进行光敏氧化时, 得到 6,14-过氧-7,8-二氢枞酸. Schuller ${ }^{[16]}$ 和 Barbara ${ }^{[17]}$ 等研究指出松香光敏氧化生 成全氧化物, 而长叶松酸的光敏氧化迅速形成跨环 过氧化物. 王素华等 ${ }^{[18]}$ 开展了对氨基苯磺酸钠的光 化学动力学研究. 但枞酸或松香的光氧化反应动力 学研究较少, 特别是具有实际应用价值的固态枞酸 和松香对光的耐候性研究没有受到足够重视.

本文首先研究枞酸的光氧化反应, 然后从实际 应用角度研究松香的光氧化反应. 为了避免枞酸或 松香在溶液中发生聚合等副反应, 设计了枞酸和松 香光氧化室进行固态的光氧化反应, 采用紫外-可见 分光光度计考察枞酸在聚乙烯膜上的紫外吸收光谱 特征峰, 测定枞酸和松香在紫外光下的氧化反应动 力学, 探索光对氧化反应的影响, 寻找光强度与反应 速率常数之间的关系, 为今后松香对光的耐候性评 价和在涂料、油墨、胶粘剂、造纸施胶剂等领域的新 产品研究开发提供理论依据.

\section{1 实验部分}

\section{1 材料与仪器}

松香(特级品), 产自广西; 枞酸 (二戈胺盐法自 制); 无水乙醇(AR, 汕头市西陇化工有限公司); 盐酸 (AR, 广东廉江市爱廉化学试剂有限公司); 二戊胺 ( $\geqslant 99$, Aldrich); 乙酸(AR, 广东光华化学有限公司).

紫外-可见分光光度仪(UV-2550, 日本岛津制作 所); 气相色谱-质谱联用仪(GC-MS/QP5050A, 日本 岛津制作所); 紫外强度检测仪(ZQJ-254 型, 上海宝 山顾村电光仪器厂).

\section{2 实验步骤和方法}

\section{2 .1 枞酸制备 $^{[19}$}

取松香 $100 \mathrm{~g}$, 经异构化反应, 再用有机胺盐结 晶得到枞酸 $50 \mathrm{~g}$, 用气相色谱-质谱联用仪定量分 析, 枞酸含量为 $97.8 \%$.

\section{2 .2 聚乙烯膜微量反应器}

将铝薄片分割成相同的两块长方形薄板, 每块
板按紫外-可见分光光度仪光路通道的位置和尺寸 开一个 $3.0 \mathrm{~cm} \times 1.7 \mathrm{~cm}$ 长方形小孔, 把聚乙烯膜夹在 其中固定.

\section{2 .3 枞酸光氧化室设计}

枞酸光氧化室由恒温干燥箱改造而成, 把紫外 灯固定在顶部, 将枞酸样品置于紫外灯的正下方, 用 紫外光强度测定仪测定紫外光的强度, 通过改变样 品与紫外灯的距离, 调节紫外光强度. 实验在空调房 进行, 保持室内的湿度基本恒定.

\section{2 .4 枞酸光氧化}

准确称取枞酸 $0.4000 \mathrm{~g}$, 倒人 $10 \mathrm{~mL}$ 的容量瓶, 用无水乙醇溶解, 定容至刻度得到枞酸溶液. 用微量 进样器准确量取枞酸溶液 $20 \mu \mathrm{L}$ 均匀滴加到微量反 应器薄膜上, 将反应器置于光氧化室中进行氧化实 验, 用紫外光谱(UV)实时检测其氧化过程.

\section{3 实验原理}

光氧化动力学就是在光的作用下进行的化学反 应动力学 ${ }^{[20]}$, 它有许多特殊的规律. 枞酸在紫外光照 射条件下, 氧化反应如下所示:

(1) 光氧化反应

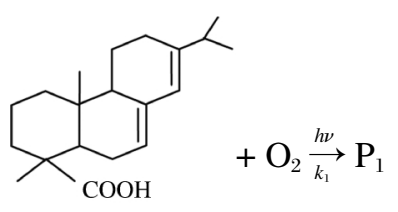

(2) 热氧化反应

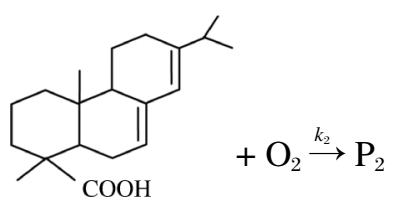

枞酸的氧化反应动力学表示式为:

$v=-\mathrm{d} n_{\mathrm{A}} / \mathrm{d} t=-k_{1} n_{\mathrm{A}}^{\alpha_{1}} n_{\mathrm{B}}^{\beta_{1}}-k_{2} n_{\mathrm{A}}^{\alpha_{1}} n_{\mathrm{B}}^{\beta_{2}}$

式中, $v$ 为枞酸的氧化速率, $k_{1}$ 为光氧化的反应速率 常数, $k_{2}$ 为热氧化的反应速率常数, $n_{\mathrm{A}}$ 和 $n_{\mathrm{B}}$ 分别表 示枞酸和氧的摩尔数, $\alpha_{1}$ 和 $\alpha_{2}$ 分别表示枞酸光氧化 和热氧化的反应级数, $\beta_{1}$ 和 $\beta_{2}$ 分别为氧在光氧化和 热氧化的反应级数. 对于开放体系, 氧气量为过量, $n_{\mathrm{B}}^{\beta_{1}}$ 和 $n_{\mathrm{B}}^{\beta_{\mathrm{B}}}$ 为常量, 故表达式可简化为:

$$
-\mathrm{d} n_{\mathrm{A}} / \mathrm{d} t=-\left(k_{1 \mathrm{~b}} n_{\mathrm{A}}^{\alpha_{1}}+k_{2 b} b_{\mathrm{A}}^{\alpha_{2}}\right)
$$

其中 $k_{1 \mathrm{~b}}=k_{1} n_{\mathrm{B}}^{\beta_{1}}, k_{2 b}=k_{2} n_{\mathrm{B}}^{\beta_{\mathrm{B}}}$. 上式中的 $k_{1 \mathrm{~b}}$ 为光氧化表观动 力学常数, $k_{2 \mathrm{~b}}$ 为热氧化表观动力学常数. 由于枞酸 的热氧化动力学是表观一级反应 ${ }^{[8]}$, 另外实验数据表 明, 光氧化反应的浓度变化值大大于热氧化反应的 浓度变化值, 即 $k_{\mathrm{lb}}$ 大大于 $k_{2 \mathrm{~b}}$ 时, 热氧化反应部分可 
以忽略, 只需考察光氧化动力学即可. 假设枞酸的光 氧化反应也为一级反应, 即 $\alpha_{1}=\alpha_{2}=1$, 则式(2)为:

$$
-\mathrm{d} n_{\mathrm{A}} / \mathrm{d} t=-\left(k_{1 \mathrm{~b}}+k_{2 \mathrm{~b}}\right) n_{\mathrm{A}}
$$

令 $k_{\mathrm{b}}=k_{1 \mathrm{~b}}+k_{2 \mathrm{~b}}$, 则式(3)变为:

$$
-\mathrm{d} n_{\mathrm{A}} / \mathrm{d} t=-k_{\mathrm{b}} n_{\mathrm{A}}
$$

\section{4 枞酸及其氧化物的定量分析方法 ${ }^{[8,21]}$}

由于枞酸有共轭双键, 在波长 $242 \mathrm{~nm}$ 处有最 大吸收峰, 枞酸氧化物在该波长下没有显著紫外吸 收, 枞酸在聚乙烯膜上定量分析的工作曲线方程 ${ }^{[8]}$ 为: $y_{1}=1.149 \times 10^{6} x_{1}+0.6365\left(y_{1}\right.$ 为吸光度, $x_{1}$ 为枞酸物 质的量, 单位 $\mathrm{mol}$ ), 枞酸氧化物工作曲线方程为: $y_{2}=$ $3.944 \times 10^{5} x_{2}-0.487\left(y_{2}\right.$ 为吸光度, $x_{2}$ 枞酸氧化物物质 的量, 单位 mol), 利用枞酸或枞酸氧化物物质的量 与吸光度关系, 通过测定吸光度来计算枞酸或枞酸 氧化物的物质的量. 由于在相同浓度下枞酸氧化物 吸光度仅为枞酸的吸光度的 $7 \%$, 在进行动力学数 据处理时, 进行如下近似处理.

设枞酸的初始物质的量为 $x_{0}$, 氧化反应进行到 $t$ 时刻, 测定的 UV 总吸光度值为 $y_{1 t}$ (枞酸和枞酸氧化 物吸光度之和), 首先把 $y_{1 t}$ 作为枞酸的吸光度值, 利 用吸光度与枞酸的物质的量工作曲线方程计算 $t$ 时 刻枞酸的物质的量 $\left(x_{1 t}\right)$, 得到 $t$ 时间枞酸反应消耗的 物质的量为 $\left(x_{0}-x_{1 t}\right)$, 由于枞酸反应物质的量等于枞 酸氧化物生成物质的量 $\left(x_{2 t}\right)$, 即 $x_{2 t}=\left(x_{0}-x_{1 t}\right)$, 根据枞酸 氧化物的吸光度与物质的量工作曲线方程计算相应 的枞酸氧化物吸光度 $y_{2 t}$, 于是枞酸吸光度 $y_{1 t}^{1}=\left(y_{1 t}-\right.$ $y_{2 t}$ ), 再利用 $y_{1}$ 和 $x_{1}$ 关系计算枞酸 $t$ 时刻的物质的量 $x_{1 t}^{1}$. 虽然, 该方法带来一定误差, 但由于枞酸氧化物 的吸光度比枞酸的吸光度小很多, 误差很小. 另外, 采用迭代法思路, 上述得到计算结果再重复上述步 骤计算一次, 两次的计算值很接近.

\section{2 结果与讨论}

\section{1 枞酸的光氧化动力学}

将枞酸置于温度分别为 $20 、 25 、 35{ }^{\circ} \mathrm{C}$, 光强度分 别为 $1100 、 660 、 600 、 420 、 400 、 260$ 和 $250 \mu \mathrm{W} \cdot \mathrm{cm}^{-2}$ 的光氧化室下进行光氧化反应. 在同一温度和光强 度下, 测定不同反应时间枞酸的 UV 吸光度, 经动力 学数据处理得到枞酸物质的量 $n$, 作 $\ln n$ 与时间 $t$ 的 曲线(图 1). 由图 1 看出, 枞酸 $\ln n$ 与时间 $t$ 均为线性 关系, 说明假定枞酸在紫外光辐照下的氧化反应为 表观一级反应是合理的. 它们在 $20 、 25$ 和 $35{ }^{\circ} \mathrm{C}$ 下的 反应动力学速率常数如表 1 所示. 由表 1 看出, 紫外 光照射引发的枞酸氧化速率常数比无紫外光照射时 的热氧化速率常数大很多, 而且, 光强度越大, 速率 常数增加幅度越大.

\section{2 枞酸的光量子产率}

为了表示反应物分子数与被吸收光子数之间的 关系, 即表达光化学反应的效率, 有必要引人量子产 率, 根据光量子产率的定义 ${ }^{[22]}$, 量子产率 $(\Phi)$ 为在一 定时间内反应的物质的量与同一时间被吸收的爱因 斯坦数的比值. 根据化学反应动力学可以计算反应 的物质的量, 即 $-\mathrm{d} n_{\mathrm{A}} / \mathrm{d} t=-k_{\mathrm{b}} n_{\mathrm{A}}$. 利用测定的枞酸吸收 紫外光可以计算被吸收的爱因斯坦数 $R(t)$, 即 $R(t)=$ $\left(I_{0}-I_{1}\right) S /\left(N_{0} h \nu V\right)$, 其中, $I_{0}$ 为紫外光照射到枞酸样品 的光强度, $I_{1}$ 为通过样品后的光强度, $S$ 为照射枞酸 样品面积, $N_{0}$ 为阿佛加德罗常数, $h$ 为普朗克常数, $\nu$ 为照射紫外光频率 $(\nu=1 / \lambda, \lambda$ 为波长), $V$ 为枞酸样 品的体积. 因此, 枞酸的光量子产率为:

$$
\Phi=\left(-\mathrm{d} n_{\mathrm{A}} / \mathrm{d} t\right) / R(t)
$$

实验中, 调节光源到枞酸的距离, 测定其光强度 $I$, 然后测定通过聚乙烯膜的光强度 $I_{0}$, 把枞酸附到 聚乙烯膜后测定通过样品的光强度 $I_{1}, S=5.1 \mathrm{~cm}^{2}$, $V=7.5 \times 10^{-4} \mathrm{~cm}^{3}$, 初始浓度 $C_{0}=2.65 \times 10^{-6} \mathrm{~mol} \cdot \mathrm{L}^{-1}$, 紫
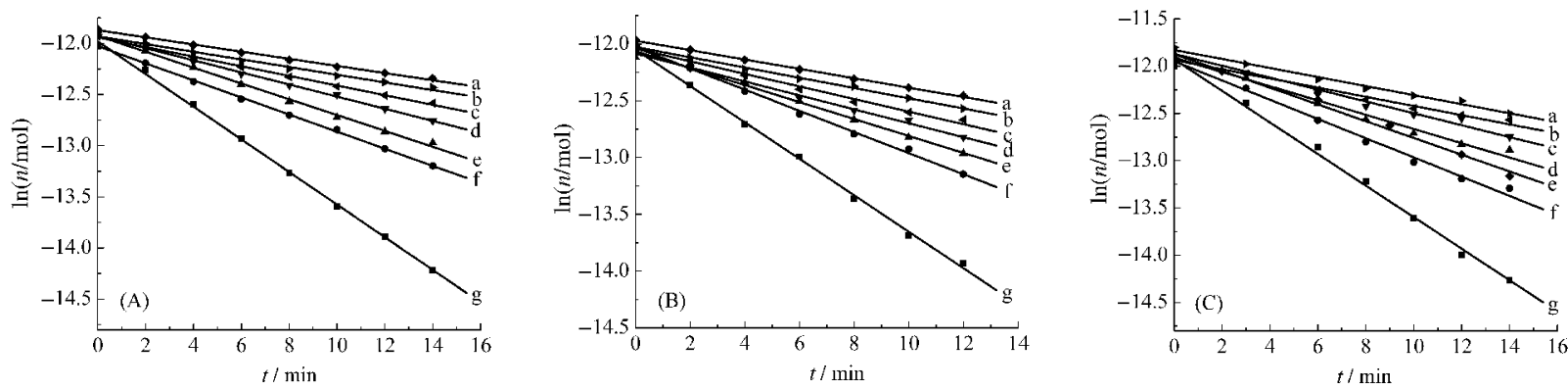

图 1 不同光强度下枞酸氧化 $\ln n$ 与 $t$ 的关系

Fig.1 Relationship between $\ln n$ and $t$ of abietic acid oxidation under different light intensities $n$ : molar number of abietic acid; $I /\left(\mu \mathrm{W} \cdot \mathrm{cm}^{-2}\right)$ : (a) 250, (b) 260, (c) 400, (d) 420, (e) 600, (f) 660, (g) 1100; (A): $20{ }^{\circ} \mathrm{C}$, (B) $25{ }^{\circ} \mathrm{C}$, (C) $35{ }^{\circ} \mathrm{C}$ 
表 1 不同光强度下枞酸氧化动力学常数 $\left(k_{\mathrm{b}}\right)$

Table 1 Rate constants $\left(k_{\mathrm{b}}\right)$ of abietic acid oxidation under different light intensities

\begin{tabular}{cccc}
\hline \multirow{2}{*}{$I /\left(\mu \mathrm{W} \cdot \mathrm{cm}^{-2}\right)$} & \multicolumn{3}{c}{$k_{\downarrow} / \mathrm{min}^{-1}$} \\
\cline { 2 - 4 } & $20{ }^{\circ} \mathrm{C}$ & $25{ }^{\circ} \mathrm{C}$ & $35{ }^{\circ} \mathrm{C}$ \\
\hline 1100 & 0.1596 & 0.1607 & 0.1671 \\
660 & 0.0836 & 0.0932 & 0.1018 \\
600 & 0.0774 & 0.0801 & 0.0937 \\
420 & 0.0593 & 0.0614 & 0.0754 \\
400 & 0.0471 & 0.0554 & 0.0629 \\
260 & 0.0375 & 0.0452 & 0.0486 \\
250 & 0.0349 & 0.0413 & 0.0479 \\
no UV light ${ }^{[8]}$ & 0.0017 & 0.0023 & 0.0041 \\
\hline
\end{tabular}

外灯的波长 $\lambda=254 \mathrm{~nm}$, 计算得到枞酸的初期光量子 产率结果如表 2 所示.

由表 2 看出, 在实验范围内光强度对枞酸的初 期光量子产率没有显著影响, $\Phi$ 的平均值为 0.471 . 进一步研究发现, 紫外光照射枞酸样品在开始时, 紫 外光几乎全部被样品吸收, 随着时间延长, 吸收率减 小, 当调节光距离使光强度为 $660 \mu \mathrm{W} \cdot \mathrm{cm}^{-2}\left(I_{0}=600\right.$ $\left.\mu \mathrm{W} \cdot \mathrm{cm}^{-2}\right)$ 时, 不同反应时间的光量子产率如表 3 所 示.

由表 3 看出, 反应初始阶段枞酸共轭双键很容 易吸收紫外光, 形成激发态的枞酸, 因此, 光量子产 率 $\Phi$ 较大, 随着反应时间延长, 由于氧化物在表面 覆盖, 阻碍反应深人内部进行氧化反应, 从而 $\Phi$ 显 著减小.

\section{3 枞酸光氧化动力学活化能的计算}

枞酸的光氧化反应为表观一级反应动力学, 活 化能的计算利用下列公式 ${ }^{[23]}$ :

反应速率: $v=n_{0}(\mathrm{~d} \eta / \mathrm{d} t)=k f(\eta)$

将上式积分, 得: $n_{0} \int_{0}^{\eta} \mathrm{d} \eta / f(\eta)=k \int_{0}^{t} \mathrm{~d} t=k t$

表 2 光强度对枞酸的初期光量子产率的影响

Table 2 Effect of different light intensities on initial light quantum efficiency of abietic acid

\begin{tabular}{|c|c|c|c|c|c|}
\hline$\frac{I}{\left(\mu \mathrm{W} \cdot \mathrm{cm}^{-2}\right)}$ & $k_{\mathrm{b}} / \min ^{-1}$ & $\frac{I_{0}}{\left(\mu \mathrm{W} \cdot \mathrm{cm}^{-2}\right)}$ & $\frac{I_{1}}{\left(\mu \mathrm{W} \cdot \mathrm{cm}^{-2}\right)}$ & $\frac{\left(I_{0}-I_{1}\right)}{\left(\mu \mathrm{W} \cdot \mathrm{cm}^{-2}\right)}$ & $\Phi$ \\
\hline 1100 & 0.1596 & 1020 & 10 & 1010 & 0.497 \\
\hline 660 & 0.0836 & 600 & 10 & 590 & 0.446 \\
\hline 600 & 0.0774 & 550 & 10 & 540 & 0.451 \\
\hline 420 & 0.0593 & 380 & 8 & 372 & 0.501 \\
\hline 400 & 0.0471 & 355 & 8 & 347 & 0.433 \\
\hline 260 & 0.0375 & 245 & 5 & 240 & 0.491 \\
\hline 250 & 0.0349 & 235 & 5 & 230 & 0.477 \\
\hline
\end{tabular}

$I$ : light intensity on the surface of film, $I_{0}$ : light intensity of passing through film, $I_{1}$ : light intensity of passing through sample, $\Phi$ : quantum efficiency; $T=20{ }^{\circ} \mathrm{C}$
表 $320^{\circ} \mathrm{C}$ 时反应时间对枞酸光量子产率的影响

Table 3 Effect of reaction time on light quantum efficiency of abietic acid at $20^{\circ} \mathrm{C}$

\begin{tabular}{rccc}
\hline$t / \mathrm{min}$ & $I_{1} /\left(\mu \mathrm{W} \cdot \mathrm{cm}^{-2}\right)$ & $\left(I_{0}-I_{1}\right) /\left(\mu \mathrm{W} \cdot \mathrm{cm}^{-2}\right)$ & $\Phi$ \\
\hline 0 & 10 & 590 & 0.446 \\
5 & 30 & 570 & 0.304 \\
10 & 60 & 540 & 0.211 \\
15 & 100 & 500 & 0.150 \\
20 & 120 & 480 & 0.103 \\
25 & 140 & 460 & 0.0708 \\
30 & 155 & 445 & 0.0482 \\
35 & 170 & 430 & 0.0328 \\
40 & 195 & 405 & 0.0229 \\
45 & 195 & 405 & 0.0151 \\
\hline
\end{tabular}

式中, $\eta$ 为枞酸氧化的转化率, $t$ 为氧化的时间. 在一 定温度和转化率范围内, 假设 $f(\eta)$ 不随温度变化, 则

$$
\begin{aligned}
& \ln \left(t_{1} / t_{2}\right)=E_{\mathrm{a}}\left(T_{1}^{-1}-T_{2}^{-1}\right) / R=\ln \left(k_{2} / k_{1}\right) \\
& E_{\mathrm{a}}=R \ln \left(t_{1} / t_{2}\right) /\left(T_{1}^{-1}-T_{2}^{-1}\right)
\end{aligned}
$$

由式(8)可知, 只要由实验求得不同温度下的 $\eta-t$ 曲 线, 利用 25 和 $35{ }^{\circ} \mathrm{C}$ 下反应的 $\ln n$ 与时间 $t$ 关系, 取 不同温度下相同的 $\eta$, 找到对应的 $t$, 代人公式 (8)计 算 $E_{\mathrm{a}}$, 不同光强度下的活化能如表 4 所示.

由表 4 看出, 光强度越大, 枞酸氧化反应活化能 越小, 说明紫外光容易引发氧化反应. 利用表 4 数 据, 进行活化能与光强度对数关联, 结果得到线性方 程: $E_{\mathrm{a}}=-7.549 \ln I+60.02$.

\section{4 光强度和速率常数的关系}

由表 1 看出, 枞酸在一定温度下, 光强度越大, 表观速率常数 $k_{\mathrm{b}}$ 越大, 为考察光强度对 $k_{\mathrm{b}}$ 的影响, 分别作 $20 、 25$ 和 $35{ }^{\circ} \mathrm{C}$ 的 $\ln k_{\mathrm{b}}-\ln I$ 关系曲线, 结果如 图 2 所示.

从图 2 看出, $\ln k_{\mathrm{b}}$ 与 $\ln I$ 呈线性关系. $20 、 25$ 和 $35{ }^{\circ} \mathrm{C}$ 下的线性方程分别为:

$$
\begin{aligned}
& \ln k_{\mathrm{b}}=0.991 \ln I-8.860 \\
& \ln k_{\mathrm{b}}=0.8786 \ln I-8.069 \\
& \ln k_{\mathrm{b}}=0.8364 \ln I-7.690
\end{aligned}
$$

\section{5 松香的光氧化动力学}

松香是由枞酸、长叶松酸、海松酸等结构相似化 合物组成的混合物 ${ }^{[24-25]}$, 为了研究松香对光的耐候

\section{表 4 不同光强度下枞酸氧化反应的活化能}

Table 4 Activation energies of oxidation reaction of abietic acid under different light intensities

\begin{tabular}{cccccccc}
\hline$I /\left(\mu \mathrm{W} \cdot \mathrm{cm}^{-2}\right)$ & 250 & 260 & 400 & 420 & 600 & 660 & 1100 \\
\hline$E_{\mathrm{a}}\left(\mathrm{kJ} \cdot \mathrm{mol}^{-1}\right)$ & 19.80 & 16.57 & 15.31 & 14.53 & 11.01 & 10.54 & 7.72
\end{tabular}




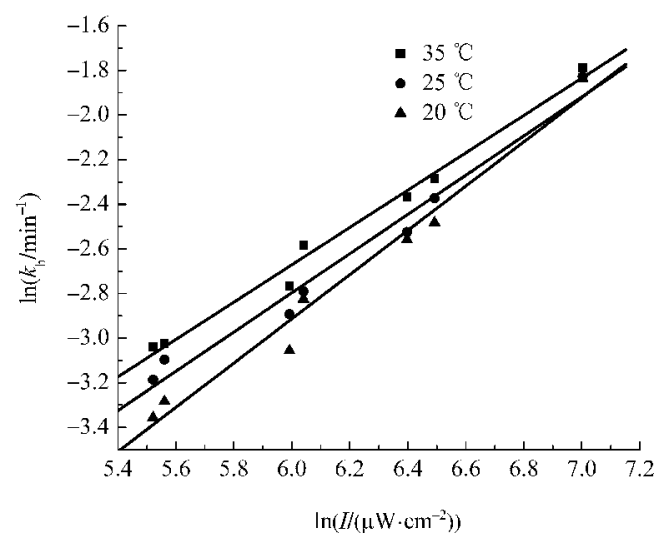

图 2 不同温度下枞酸氧化反应 $\ln k_{\mathrm{b}}$ 与 $\ln I$ 的关系

Fig.2 Plots of $\ln k_{\mathrm{b}} v s \ln I$ of oxidation reaction of abietic acid at different temperatures

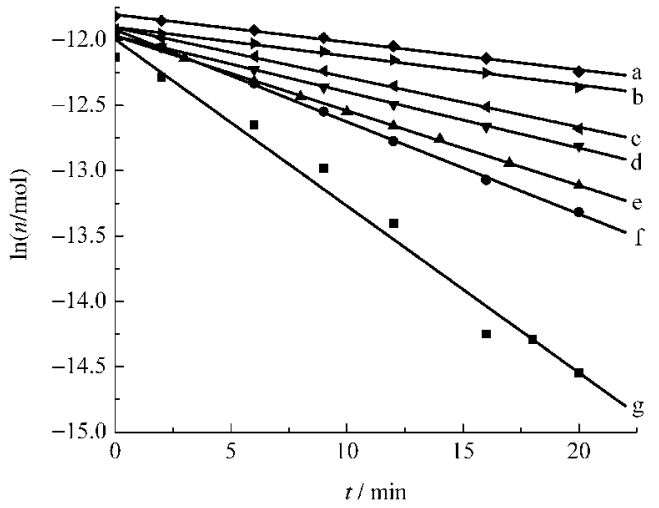

图 3 不同光强度下松香氧化 $\ln n$ 与 $\boldsymbol{t}$ 的关系

Fig.3 Relationship between $\ln n$ and $t$ of rosin oxidation under different light intensities $I /\left(\mu \mathrm{W} \cdot \mathrm{cm}^{-2}\right)$ : (a) 250; (b) 260 ; (c) 400; (d) 420; (e) 600; (f) $660 ;(\mathrm{g}) 1100$

性, 有必要在研究枞酸光氧化反应动力学基础上, 考察松香光氧化反应动力学. $20{ }^{\circ} \mathrm{C}$ 时, 不同紫外光 强度下松香氧化反应的 $\ln n$ 与时间 $t$ 的关系曲线如 图 3 所示.

由图 3 看出, 松香物质的量的 $\ln n$ 与时间 $t$ 的 关系均为线性关系, 说明了松香的光氧化反应也是 表观一级反应. 不同光强度下对应的反应动力学速 率常数如表 5 所示.

利用表 5 作 $\ln k_{\mathrm{b}}-\ln I$ 关系曲线, 发现呈线性关 系, 线性方程为: $\ln k_{\mathrm{b}}=1.204 \ln I-10.49$. 比较表 1 和表

\section{表 5 不同光强度下松香氧化速率常数}

Table 5 Rate constant of rosin under different light intensities

\begin{tabular}{ccccccccc}
\hline$\frac{I}{\left(\mu \mathrm{W} \cdot \mathrm{cm}^{-2}\right)}$ & 1100 & 660 & 600 & 420 & 400 & 260 & 250 & $\begin{array}{c}\text { no UV } \\
\text { light }\end{array}$ \\
\hline$k_{\triangleright} / \mathrm{min}^{-1}$ & 0.1275 & 0.0705 & 0.0572 & 0.0428 & 0.0380 & 0.0221 & 0.0211 & 0.0035 \\
\hline
\end{tabular}

5 , 结果发现松香氧化速率常数比枞酸氧化速率常数 小, 这是因为松香中不容易发生氧化反应的海松酸 等组分对枞酸起保护作用, 阻碍氧与枞酸进行氧化 反应。

\section{3 结 论}

通过设计固态枞酸和松香光氧化室, 实时测定 了它们的光氧化反应过程, 得出了如下结论:

(1) 枞酸和松香在紫外光辐照下的光氧化反应 动力学均呈现表观一级反应.

(2) 在紫外光强度分别 $1100 、 660 、 600 、 420 、 400 、$ 260 和 $250 \mu \mathrm{W} \cdot \mathrm{cm}^{-2}$ 下, 枞酸于 $20 、 25 、 35^{\circ} \mathrm{C}$ 的光氧化 速率常数与光强度的关系分别为: $\ln k_{\mathrm{b}}=0.9911 \ln I-$ $8.860, \ln k_{\mathrm{b}}=0.8786 \ln I-8.069, \ln k_{\mathrm{b}}=0.8364 \ln I-7.690$.

(3) $20{ }^{\circ} \mathrm{C}$ 时, 在紫外光强度分别 $1100 、 660 、 600 、$ $420 、 400 、 260$ 和 $250 \mu \mathrm{W} \cdot \mathrm{cm}^{-2}$ 下, 枞酸初始反应的 光量子产率 $\Phi$ 为 0.471 .

(4) 枞酸在不同光强度下的活化能与不同光强 度的对数为线性关系, 其线性方程为: $E_{\mathrm{a}}=-7.549 \ln I+$ 60.02 .

(5) $20{ }^{\circ} \mathrm{C}$ 下紫外光强度为 $1100 、 660 、 600 、 420$ $400 、 260$ 和 $250 \mu \mathrm{W} \cdot \mathrm{cm}^{-2}$ 时, 松香氧化速率常数与 光强度关系为: $\ln k_{\mathrm{b}}=1.204 \ln I-10.49$.

\section{References}

1 Song, Z. Q. Chemistry and Industry of Forest Products, 2004, 24 (supplement): 7 [宋湛谦. 林产化学与工业, 2004, 24(增刊): 7]

2 Zhang, Z. G.; Huang, J. M.; Kang, D. H. Journal of Nanchang University (Natural Science), 2000, 24(3): 274 [张招贵, 黄精美, 康冬华. 南昌大学学报: 理科版, 2000, 24(3): 274]

3 Chen, X. H. Chemical World, 2005, (6): 344 [陈学恒. 化学世 界, 2005, (6): 344]

4 Song, Z. Q.; Wang, Z. H.; Tang, Y. D. Fine and Specialty Chemicals, 2000, 9(18): 21 [宋湛谦, 王振洪, 唐元达. 精细与专 用化学品, 2000, 9(18): 21]

5 Zhang, G. Y. China Pulp \& Paper, 2005, 24(10): 57 [张国运. 中国造纸, 2005, 24(10): 57]

6 Harris, G. C. Org. Synth., 1952, 32(1): 1

7 Wang, T.; Su, Z. A. Chemistry \& Industry of Forest Products, 1991, 11(3): 173 [王＼cjkstart涛, 粟子安. 林产化学与工业, 1991, 11 (3): 173]

8 Qin, R. X.; Huang, P. X.; Liu, X. M.; Ma, L.; Wu, Y. L. Chem. J. Chin. Univ., 2009, 30(5): 954 [秦荣秀, 黄品鲜, 刘雄民, 马 丽, 吴晏玲. 高等学校化学学报, 2009, 30(5): 954]

9 Muthuramu, K.; Ramamurthy, V. J. Org. Chem., 1982, 47(10) 3976 
10 Li, C. M.; Hou, J.; Yu, Y.; Pan, X. X.; Hou, H. Q. Environmental Chemistry, 2000, 19(1): 18 [李朝敏, 侯 健, 于 勇, 潘循晳, 侯惠奇. 环境化学, 2000, 19(1): 18]

11 Zhang, J. C.; Wang, D. Y. Modern photochemistry. Beijing: Chemical Industry Press, 2006: 137-255［张建成, 王夺元. 现代 光化学. 北京: 化学工业出版社, 2006: 137-255]

12 Song, W.; Jiang, L. P.; Wang, Y.; Li, R. Q. Journal of Tianjin University, 2004, 37(11): 959 [宋 威, 蒋龙平, 王 颖, 李润卿. 天津大学学报, 2004, 37(11): 959]

13 Moore, R. N.; Lawrence, R. V. J. Am. Chem. Soc., 1958, 80(6): 1438

14 Moore, R. N.; Lawrence, R. V. J. Am. Chem. Soc., 1960, 82(7): 1734

15 Moore, R. N.; Lawrence, R. V. J. Am. Chem. Soc., 1961, 83(10): 2563

16 Schuller, W. H.; Minor, J. C.; Lawrence, R. V. Ind. Eng. Chem. Prod. Res. Dev., 1964, 3(2): 97

17 Barbara, G.; Ana, M. L.; Joao, M. C.; Sundaresan, P.; Henry, S. R. Tetrahedron Computer Methodology, 1988, 1(2): 133

18 Wang, S. H.; Chen, D. W. Acta Phys. -Chim. Sin., 1998, 14(5):
458 [王素华, 陈德文. 物理化学学报, 1998, 14(5): 458]

19 Harris, G. C.; Sanderson, T. F. J. Am. Chem. Soc., 1948, 70(1): 334

20 Liu, S. C. Advanced physics chemistry. Zhengzhou: Zhengzhou Univercity Press, 2005: 35-37 [刘寿长. 高等物理化学. 郑州: 郑州大学出版社, 2005: 35-37]

21 Qin, R. X.; Liu, X. M.; Ma, L.; Wu, Y. L. Applied Chemical Industry, 2008, 37(11): 1381 [秦荣秀, 刘雄民, 马 丽, 吴晏玲. 应用化工, 2008, 37(11): 1381]

$22 \mathrm{Xu}, \mathrm{Y}$. Chemical reaction kinetics. Beijing: Chemical Industry Press, 2005: 203-205 [许 越. 化学反应动力学. 北京: 化学工 业出版社, 2005: 203-205]

23 Cui, W. Q.; Feng, L. R.; Xu, C. H.; Lü, S. J.; Qiu, F. L. Acta Chim Sin., 2005, 63(3): 203 [崔文权, 冯良荣, 徐成华, 吕绍洁, 邱发 礼. 化学学报, 2005, 63(3): 203]

24 Song, Z. Q. Journal of Chemical Industry of Forest Products (Bimonthly), 2002, 36(4): 29 [宋湛谦. 林产化工通讯, 2002, 36 (4): 29]

25 Wang, S. F. Chemistry and Industry of Forest Products, 2007, 27 (32): 31 [王石发. 林产化学与工业, 2007, 27(32): 31] 